\title{
A constitutive equation for ceramic materials used in lightweight armors
}

\author{
D. Fernández-Fdz, R. Zaera* , J. Fernández-Sáez \\ Department of Continuum Mechanics and Structural Analysis, University Carlos III of Madrid, Avda. de la Universidad, 30,28911 Leganés, Madrid, Spain
}

\begin{abstract}
A B S T R A C T
A constitutive model to simulate the behavior of ceramic materials under impact loading is proposed in order to achieve a better representation of the damage process due to the material fragmentation. To integrate the proposed constitutive equations, a semi implicit algorithm (implicit for the stresses and explicit for the damage variable) has been developed, leading to the generalized expressions of the clas sical return mapping algorithm. The model was implemented in a commercial finite element code and its performance was demonstrated by comparing its predictions with experimental results obtained by other authors.
\end{abstract}

Ceramic

Constitutive equation

Return mapping algorithm

Impact

Armor

Damage

\section{Introduction}

Ceramic materials are known to provide high performance in armor applications against the impact of high speed projectiles. Used in bi layered configurations (Fig. 1) consisting of a ceramic front plate (e.g. alumina, silicon carbide, aluminum nitride, tita nium diboride) and a ductile back plate (e.g. steel, aluminum, fiber reinforced composite), such armors have attracted considerable interest since the 1960s because of their high ballistic resistance compared to monolithic metallic protections [1 7]. The light weight of these protective materials made them especially useful for applications such as body, vehicle and aircraft armor, in which lightness is a primary design requirement.

The hardness of ceramic materials causes fragmentation and erosion in the projectile. When an armor piercing projectile im pacts against a ceramic tile, the nose of the projectile is shattered or blunted, thus reducing its mass and energy. Also, the ceramic spreads the load over a larger area, the backing plate absorbing the remaining kinetic energy of the projectile by plastic deforma tion in the case of a metal alloy, or by elastic deformation and damage in case of a fiber reinforced composite.

However, as ceramics are inherently brittle, with low fracture toughness as compared to metal, they show extensive fragmenta tion after receiving an impact from a solid at high velocity. Immedi ately upon contact with the projectile, stress waves are generated in the ceramic tile and a conical cracking front propagates from the border of the projectile/ceramic contact area. When the compressive

\footnotetext{
* Corresponding author. Tel.: +34 916249983; fax: +34 916249430.

E-mail address: ramon.zaera@uc3m.es (R. Zaera).
}

wave reaches the adhesive layer at the ceramic/backing plate inter face, with lower mechanical impedance $[8,9]$, hoop tensile stresses develop at the rear face of the tile and radial cracks propagate back to the projectile. The consequence is a general break up of the ceramic from the coalescence of the cracks. Though continuing throughout the penetration process, the fragmentation that occurs in the first microseconds after impact is key in weakening the cera mic strength. Before the end of this fragmentation stage, the ceramic material along the path of the projectile deforms essentially in a uni axial strain condition, and thus the material shows its greatest strength against being pierced and the tip of the projectile is strongly eroded.

After fragmentation, the projectile penetrates a conoid of com minuted ceramic, the mechanical properties of which are lower than those of the undamaged tile and depend on the degree of frag mentation at each point. Crack generation as described above allows the displacement of small fragments, facilitating projectile penetration. However, the erosion of the projectile tip may con tinue during this phase. The volume of fragmented ceramic ahead of the projectile, physically separated from the remaining intact ceramic tile, has a conoid shape. The conoid is accelerated in the im pact direction and distributes the load over a large area of the back ing panel which undergoes extensive deformation, contributing greatly to increase the ballistic performance of the ceramic faced armor. Although ceramic materials are brittle, they can have signif icant strength after failure under confined conditions. The strength of both the intact and failed materials increase with the confining pressure. Heard and Cline [10] analyzed the behavior of various types of confined ceramics and showed that, at high confining pres sures, the material undergoes a brittle to ductile transition and the ceramic subsequently deformed in a stable manner. 


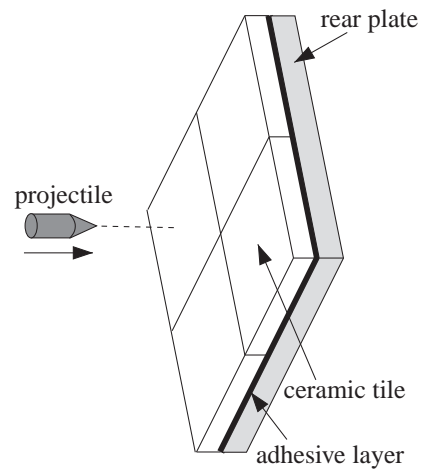

Fig. 1. Layout of a ceramic-faced lightweight armor.

Finite element, finite differences, or meshless codes have pro ven to be valuable tools for analyzing and designing ceramic faced armor. A number of numerical codes are commercially available, and these are successful in simulating high speed impact prob lems. However, their capacity for close approximation to reality depends heavily on the constitutive equations used for the materi als. The main problem with these models is the uncertainty with regard to the input data required for the materials, requiring that the model be calibrated for each material to be used. Moreover, not every code provides constitutive equations for the modeling of ceramics under shock and impact, and thus users often need to implement specific features in order to model the behavior of the material.

Several constitutive relations have been proposed in the last two decades to model the dynamic fragmentation undergone by a ceramic tile, most of them based on a continuum damage ap proach. Following a chronological order, we can firstly find the work due to Curran et al. [11], called BFRACT 3, which considers a statistical distribution of plane and circular cracks in the ceramic, growing at a rate given by an exponential law of the stress normal to the flaw. One of the most widely used constitutive models for simulating the post yield response of brittle materials to ballistic impacts are those due to Johnson and Holmquist in the 1990s. The first version of the model, called the JH 1 [12], was developed to account for large deformations of brittle materials such as ceramics, rocks and concrete over a range of strain rates. The sec ond version, called JH 2 [13], took into consideration progressive degradation with increasing deformation through a damage evolu tion rule. Fahrenthold [14] developed a continuum model in which damage is described by a second order tensor. A stress based dam age evolution law and a failure criterion based on a critical level of damage were used. Fahrenthold [14] used the model to calculate the depth of penetration in a steel plate impacted by a sphere of alumina. Cortés et al. [15] proposed a model which considers a sca lar damage variable representing the degree of fragmentation of the ceramic. Hydrostatic stress is assumed to be the governing var iable for damage nucleation and growth. Then, the yield stress is calculated with a direct mixture law, averaging a Drucker Prager criterion for intact ceramic fraction and a frictional criterion for the comminuted ceramic fraction, the damage being the weight factor. Rajendran and Grove $[16,17]$ proposed an internal state var iable based constitutive model for ceramic materials, which incor porated micro crack propagation under both compression and tension, and void collapse due to the plastic flow in the matrix sur rounding the pores. The micro crack damage is described using a dimensionless damage density parameter in terms of the maxi mum micro crack size and the average number of micro cracks per unit volume. The damage evolution in terms of crack growth is formulated based on a generalized Griffith criterion. This model has been implemented in commercial FE codes and also in meshless methods [18] to describe the response of ceramics sub jected to various stress/strain loading conditions, including impacts. Simha et al. [19] proposed a phenomenological approach to model the constitutive behavior of the ceramic material under impact. Progressive fragmentation of the material is defined through a scalar damage variable, which is used to determine the compressive strength. The yield surface is defined as in conven tional metal plasticity and the Mie Gruneisen equation of state is used. Together with and element removal scheme for ceramics, the model is used to investigate the penetration response of AD 99.5 alumina in the depth of penetration test. Bar on et al. [20] presented a model which uses phenomenological evolution equations, both for fracture mechanics criteria considering pen ny shaped cracks and for pore compaction and dilation. The consti tutive equations are determined by satisfying the thermodynamics principles, after proposing a functional form for the Helmholtz free energy. The ability of the model to capture the response of ceramic to dynamic shock loading were demonstrated by the simulation of a dynamic plate impact experiment on alumina. Zuo et al. [21] developed the Dominant Crack Model (DCA), a rate dependent, continuum damage model for the dynamic response of brittle materials. A damage surface is proposed by applying the general ized Griffith instability criterion to the dominant crack having the most unstable orientation. The evolution of damage is based on the energy release rate for the dominant crack, thus giving a physical basis for damage growth. Moreover the model incorpo rates crack opening as a source of volume increase due to shear (dilatancy). The performance of the model was validated through numerical results of a silicon carbide under several loading paths and strain rates. Deshpande and Evans [22] presented a mecha nism based constitutive model, which comprises micro crack extension rates based on stress intensity calculations, effect of the crack density on the stiffness, and plasticity at high confining pressures. Additionally, it considers the effect of microstructural parameters by taking into account initial flaws that scale with the grain size. The model is able to capture the transition from deformation by micro cracking at low triaxiality to plastic slip at high triaxialities.

Besides the continuum damage models, other authors were interested in predicting the discrete nature of cracks in brittle material. Camacho and Ortiz [23] followed the nucleation and propagation of discrete cracks along arbitrary paths by recourse to a cohesive fracture model. In the constitutive model, an equa tion of state of the Mie Gruneisen type is adopted in conjunction with power hardening law with linear thermal softening. Follow ing this seminal work, Zhou and Molinari [24] proposed a linear, homogeneous and isotropic elastic behavior for the bulk material and cohesive elements to describe the mechanical behavior of the micro cracks. The interfaces between two neighboring ele ments are treated as possible cracks, which may be activated to form the cohesive elements, and the local cohesive parameters are considered to follow a stochastic distribution. This model was used by the same workers [25] to investigate the probabilistic fail ure of a model ceramic system under dynamic tensile loading. Later on, Lee et al. [26] modeled the discrete nature for damage of brittle materials implementing a cohesive law fracture model with a node separation algorithm for the tensile failure and Mohr Coulomb model for the compressive loading. Using tetrahe dral instead of hexahedral finite elements they provided more potential fracture surfaces for crack propagation. The model was verified by simulating oblique impact into the composite metal/ ceramic systems.

Parallel to these works, several models were developed for the simulation of concrete under impact loading, sharing many fea tures with those specifically proposed for ceramic materials. For instance, Malvar et al. [27] proposed the widely used K\&C model 
which uses a mixture law to determine the yield surface of the damage material, as considered by some authors for ceramic mate rials [13,15,19]. Also Zhou and Hao [28] used a mixture law driven by a scalar damage parameter to determine the yield stress of the damaged mortar in a mesoscale model for concrete submitted to impact loading; the yield stress of both undamaged and residual strength materials were defined through a piecewise pressure dependent law. Similarly, Hentz et al. [29] considered a Mohr Coulomb criterion to reproduce the pressure dependent behavior of concrete, reducing the slope and neglecting cohesion after fail ure. The Johnson Holmquist model [13], commonly used for cera mic materials, was enhanced by Riedel and co workers [30,31], leading to the RHT model. Several new features like strain harden ing, third invariant dependent yield surface, and an independent fracture strength surface, were incorporated to allow a more appropriate modelling of concrete softening. Tu and $\mathrm{Lu}$ [32] com pared and examined in detail both RHT and K\&C models through a series of numerical tests, and presented a valuable review of dif ferent concrete material models used in hydrocodes.

The present work proposes a constitutive model for ceramics used in armor applications, with its corresponding integration algorithm and methodology to determine material parameters. Although, as it has been pointed out in the previous review, there are more complex constitutive relations to model the dynamic fragmentation undergone by the ceramic tile some of them require a large number of material parameters or large computa tional times, some were not validated with real impact tests on lightweight ceramic armors our model makes a simpler descrip tion of the fragmentation process and provides precise results in terms of the resistance of the ceramic material to be penetrated. The model, based on the one developed by Cortés et al. [15], was validated with experimental results that indicated a high predic tive capability. The algorithm may be easily implemented in numerical codes and inherits the robustness and stability of return mapping methods. The parameters of a given ceramic material may be determined with the experimental results of the depth of penetration (DOP) test, well established in the field of high speed impact protection.

\section{Constitutive relations}

The motion of a deformable body can be described through the deformation gradient tensor $F$. This tensor transforms an infinites imal material vector $d X$ into the corresponding spatial vector $d x$, i.e.

$d x \quad F d X$.

From this tensor, the following rate tensors can be defined as follows:

- the velocity gradient tensor, $l \dot{F} F{ }^{1}$,

- the rate of deformation tensor, $d \quad \frac{1}{2}\left(l+l^{T}\right)$,

- and the spin tensor, $w \quad \frac{1}{2}\left(l \quad l^{T}\right)$.

To separate the recoverable and non recoverable contributions of the deformation gradient, the Kröner Lee multiplicative split of $F$ is assumed [33,34].

F $F^{e} F^{p}$,

where $F^{e}$ and $F^{p}$ represent the reversible elastic deformation and inelastic deformation of the material, respectively. This decomposi tion implies the so called plastic intermediate configuration defined by $F^{e}{ }^{1}$, which is valid only locally. We may equally write the spatial velocity gradient as

$l \quad \dot{F} F^{1} l^{e}+F^{e} l^{p} F^{e^{1}}$, $l^{e}$ and $l^{p}$ being the elastic and plastic spatial velocity gradients de fined as

$l^{e} \quad \dot{F}^{e} F^{e^{1}}, \quad l^{p} \quad \dot{F}^{p} F^{p^{1}}$.

For ballistic applications, elastic strains (and rates) are commonly very small compared to unity or to plastic strains (and rates). With this restriction, we get the following approximations:

$F^{e} \quad U^{e} \quad V^{e} 1+\mathrm{O}\left(\varepsilon^{e}\right)$,

where $U^{e}$ and $V^{e}$ are the right and left elastic stretch tensors, and 1 is the unit second order tensor. This also implies to consider negligible rigid body rotation in $F^{e}$. Taking advantage of this, Eq. (3) leads to

$l l^{e}+l^{p}$.

Its symmetric part leads to the additive decomposition of the rate of deformation tensor $d$, generally assumed for hypoelastic plastic materials $[35,36]$

$d d^{e}+d^{p}$.

Ceramic materials used in armor, when submitted to impact, show small elastic strains and strain rates as compared to inelastic ones. With this assumption, the elastic strain rate is provided by the fol lowing expression of Hooke's law

$\sigma^{\nabla} \quad C: d^{e} \quad C:\left(\begin{array}{ll}d & d^{p}\end{array}\right)$,

where $\sigma^{\nabla}$ is an objective rate of the Cauchy stress tensor and $C$ is the Hooke stress strain tensor given by

C $2 G I_{d e v}+K 1 \otimes 1$,

where $G$ and $K$ are the elastic constants

$G \quad \frac{E}{2(1+v)}$,
$K \quad \frac{E}{3(1 \quad 2 v)}$.

$I_{\text {dev }}$ being the deviatoric projector

$I_{\text {dev }} \quad I \quad \frac{1}{3} 1 \otimes 1$

with $I$ the unit fourth order tensor exhibiting only minor symmetry

$(I)_{i j k l} \quad \delta_{i k} \delta_{j l}$

and 1 being the unit second order tensor

$(1)_{i j} \quad \delta_{i j}$

To describe the inelastic behavior of ceramic, the model takes into account the progressive fragmentation of a monolithic intact cera mic material by means of a scalar damage variable $D$, varying in the range $[0,1]$ (intact pulverized material). As proposed by Cortés et al. [15] the model considers a Drucker Prager law to define yield ing for the intact material $(D=0)$

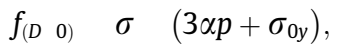

where $\bar{\sigma}$ is the equivalent stress defined as

$\sigma \quad \sqrt{\frac{3}{2}} s: s$

$s$ being the deviatoric stress tensor, and $p$ the hydrostatic pressure defined as

p $\frac{\sigma: 1}{3}$,

where $3 \alpha$ is a parameter related to the internal friction angle of the material and $\sigma_{0 y}$ is the material cohesion. For the pulverized mate rial $(D=1)$ a pure frictional law is proposed 


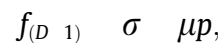

with $\mu$ being the friction coefficient. Intermediate damage states are defined as an average of the two situations, with a direct mixture law applied to the yield function, $D$ being the mixture parameter

$\left.\begin{array}{llll}f & \sigma & (1 & D\end{array}\right)\left(3 \alpha p+\sigma_{0 y}\right) \quad \mu p D$.

We proposed a damage growth law driven by the maximum princi pal stress $\sigma_{1}$

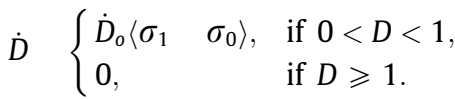

$\dot{D}_{0}$ being a material parameter related to the growth rate of the cracks, $\sigma_{0}$ being a stress threshold from which the fracture starts to develop, and \langle\rangle the MacCauley brackets. Other models for ceramics under dynamic loading also propose $\sigma_{1}$ as the variable driving fragmentation [37,38]. The maximum principal stress $\sigma_{1}$ can be determined by the closed form expression

$\sigma_{1} \quad p+\frac{2}{3} \sigma \cdot \cos \theta$

where the Lode angle $\theta$ is defined by

$\cos 3 \theta \quad \frac{3 \sqrt{ } 3}{2} \frac{J_{3}}{\sqrt{ } J_{2}^{3}}$,

where $J_{2}$ and $J_{3}$ are the second and third invariants of the deviatoric stress tensor $s$, respectively.

To describe the behavior for pressure sensitive materials, asso ciative plasticity laws are often inappropriate since they overesti mate the volumetric part of the plastic strain [39]. Therefore we have chosen a non associative plastic flow rule

$d^{p} \quad \dot{\lambda} \frac{\partial \Psi}{\partial \sigma} \quad \dot{\lambda} \Psi_{\sigma}$

where the plastic potential $\Psi$ is formally analogous to the yield function (Eq. (19)) but has a lower slope with the pressure

$\Psi \quad \sigma \quad(1 \quad D)\left(3 \alpha_{\Psi} p+\Upsilon\right) \quad \mu_{\Psi} p D$,

$\Upsilon$ being a dummy parameter, $\alpha_{\Psi}=k \alpha$ and $\mu_{\Psi}=k \mu$ for $0 \leqslant k<1$. The derivative of the potential may be expressed as

$\left.\Psi_{\sigma} \quad \frac{3}{2} \frac{s}{\sigma}+\left[\begin{array}{ll}1 & D\end{array}\right) \alpha_{\Psi}+\frac{\mu_{\Psi} D}{3}\right] 1$.

This avoids the overestimation of the dilatant effect of the frag mented ceramic material due to the inelastic deformations [37]. The decrease in the slope of the yield cone in the Haigh Westerg aard stress space due to the increased damage also includes the de crease in the dilatant behavior as the ceramic material is being fragmented.

The solution of the above equations must be subjected to the Kuhn Tucker complementary conditions

$\dot{\lambda} \geqslant 0, \quad f \leqslant 0, \quad$ if $\quad 0$

and the consistency condition

$\dot{\lambda} \dot{f} \quad$

\section{Integration of the constitutive equations}

For the integration of the above rate equations, incremental objectivity was achieved by rewriting them in a neutralized config uration [40 43]. To formalize this approach, $\varpi$ being a spatial skew symmetric tensor, we may generate a group of rotations $\mathfrak{R}$ such that

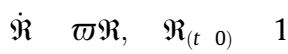

with

$\varpi \varpi^{T}$

and

$\mathfrak{R}^{1} \mathfrak{R}^{T}$

Typical choices of $\varpi$ include the spin tensor $w$ and the tensor $\Omega$ de fined as

$\Omega \quad \dot{R} R^{T}$,

where $R$ is the rotation tensor given by the polar decomposition theorem of $F$. The Cauchy stress tensor and the rate of deformation tensor are rotated as

$\sigma_{\Re} \quad \mathfrak{R}^{T} \sigma \mathfrak{R}, \quad d_{\mathfrak{R}} \quad \mathfrak{R}^{T} d \mathfrak{R}$.

We find that time differentiation of the rotated Cauchy stress leads to

$\dot{\sigma}_{\mathfrak{R}} \quad \mathfrak{R}^{T}(\dot{\sigma}+\sigma \varpi \quad \varpi \sigma) \mathfrak{R} \quad \mathfrak{R}^{T} \sigma^{\nabla} \mathfrak{R}$,

where $\sigma^{\nabla}$ coincides with the Green Naghdi McInnis stress rate if $\varpi=\Omega$ and then $\Re \quad R$, or with the Jaumann stress rate if $\varpi=w$ (see [41] for a description of the algorithm to integrate Eq. (28) in this case). Thus, a complex objective stress rate can be computed as a simple time derivative. Moreover, taking advantage of the orthogonality of $R$, the symmetry of the Cauchy stress and rate of deformation tensors and the isotropy of the elastic tensor $\left(\begin{array}{ll}C_{\Re} & C\end{array}\right)$, we find that the rate equations defined above are form identical in the rotated configuration

$\dot{\sigma}_{\Re} \quad C: d_{\Re}^{e} \quad C:\left(\begin{array}{ll}d_{\Re} & d_{\Re}^{p}\end{array}\right)$

$\sigma_{\mathfrak{R}} \quad s_{\mathfrak{R}} \quad p 1$,

$\sigma \quad \sqrt{\frac{3}{2}} s_{\Re}: s_{\Re}$,

$d_{\mathfrak{R}}^{p} \quad \dot{\lambda} \frac{\partial \Psi}{\partial \sigma_{\mathfrak{R}}}$

and scalar Eqs. (19) and (20) remain unchanged.

\subsection{Time discretization and solving algorithm}

To integrate the constitutive equations of the ceramic material we have proposed a semi implicit return mapping algorithm (ex plicit for the incremental treatment of the damage evolution (Eq. (20))). If a Newton Raphson scheme is used to solve the set of non linear equations, a complete implicit formulation would in volve the inversion of a $7 \times 7$ matrix in each subiteration, with the consequent increase of the computational cost. This is the re sults of including the maximum principal stress $\sigma_{1}$ in the equation of the damage evolution (Eq. (20)). Thus, the discretized form of this equation is written as

$D_{n+1} \quad D_{n}+\dot{D}_{0}\left\langle\sigma_{1 n} \quad \sigma_{0}\right\rangle \Delta t$

with $\Delta t$ being the time increment. Once $D_{n+1}$ has been calculated, the yield function is determined by

$f_{n+1} \quad f\left(\sigma_{\Re_{n+1}}, D_{n+1}\right) \quad 0$.

Another advantage of this semi implicit scheme is the simplicity of the return to the yield surface, achieved with no iterations, in accor dance with a Drucker Prager plasticity criterion without hardening.

Following the scheme predictor corrector, the actualized ro tated stress is given by

$\sigma_{\Re_{n+1}} \quad \sigma_{\Re_{n+1}}^{\text {trial }}+\Delta \sigma_{\Re}^{\text {ret }}$ 
where the trial stress is determined by

$\sigma_{\Re_{n+1}}^{\text {trial }} \quad \sigma_{\Re_{n}}+C:\left(\Delta \varepsilon_{\Re}\right)$

where $\sigma_{\mathfrak{R}_{n}}$ is the rotated stress in time $n$

$\sigma_{\mathfrak{R}_{n}} \quad \mathfrak{R}_{n}^{T} \sigma_{n} \Re_{n}$

and $\Delta \varepsilon_{\Re}$ is the increment of total deformation in the co rotational frame, which could be determined by an objective approximation of the rate of deformation tensor $d_{n+1 / 2}$ calculated by the midpoint rule (see $[41,42]$ )

$\Delta \varepsilon_{\Re} \quad \Delta t d_{\Re_{n+1 / 2}} \quad \Delta t \Re_{n+1 / 2}^{T} d_{n+1 / 2} \Re_{n+1 / 2}$,

thus, the correction $\Delta \sigma_{\Re}^{\text {ret }}$ to the trial stress is determined by

$\Delta \sigma_{\Re}^{r e t} \quad C:\left(\Delta \lambda \Psi_{\sigma \Re_{n+1}}\right)$

$$
\left.3 G \Delta \lambda \frac{s_{\Re_{n+1}}}{\sigma_{n+1}} \quad 3 K \Delta \lambda\left[\begin{array}{ll}
1 & D_{n+1}
\end{array}\right) \alpha_{\Psi}+\mu_{\Psi} \frac{D_{n+1}}{3}\right] 1 .
$$

Since $D_{n+1}$ is initially known, only Eqs. (39) and (44) must be solved simultaneously. Both equations are written the form of residuals $R_{1}$ and $R_{2}$ suitable for Newton Raphson iteration

$R_{1} \quad C^{1}: \Delta \sigma_{\Re}^{r e t}+\Delta \lambda \Psi_{\sigma \Re_{n+1}} \quad 0$,

$R_{2} \quad f\left(\sigma_{\Re_{n+1}}, D_{n+1}\right) \quad 0$.

Linearization of these equations (considering that $\delta \sigma_{\Re}^{\text {ret }} \quad \delta \sigma_{\Re}$ if we begin the iteration from the trial state) gives

$R_{1}^{(k+1)} \approx R_{1}^{(k)}+C^{1}: \delta \sigma_{\Re}^{(k)}+\Delta \lambda^{(k)}\left(\frac{\partial \Psi_{\sigma \Re}}{\partial \sigma_{\Re}}\right)^{(k)}: \delta \sigma_{\Re}^{(k)}$

$$
+\delta \lambda^{(k)} \Psi_{\sigma \mathfrak{R}_{n+1}}^{(k)} \quad 0
$$

$R_{2}^{(k+1)} \approx R_{2}^{(k)}+\left(\frac{\partial f}{\partial \sigma_{\Re}}\right)^{(k)}: \delta \sigma_{\Re}^{(k)} \quad 0$,

$k$ being the subiteration index. These equations give $\delta \sigma_{\Re}^{(k)}$ and $\delta \lambda^{(k)}$. Solving for the plastic multiplier increment

$\delta \lambda^{(k)} \frac{R_{2}^{(k)}\left(\frac{\partial f}{\partial \sigma_{\Re}}\right)^{(k)}: T^{(k)}: R_{1}^{(k)}}{\left(\frac{\partial f}{\partial \sigma_{\Re}}\right)^{(k)}: \mathrm{T}^{(k)}: \Psi_{\sigma \Re_{n+1}}^{(k)}}$,

with $\mathrm{T}^{1}$ being the fourth order tensor

$\mathrm{T}^{1} \quad C^{1}+\Delta \lambda \frac{\partial \Psi_{\sigma \Re}}{\partial \sigma_{\Re}}$.

From Eq. (25) we see that the return direction remains constant during the iteration, so that Eq. (45) is lineal and $R_{1}^{(k)}$ expression (49) is written as

$\delta \lambda^{(k)} \frac{f_{n+1}^{(k)}}{\left(\frac{\partial f}{\partial \sigma_{\Re}}\right)^{(k)}: T^{(k)}: \Psi_{\sigma \Re_{n+1}}^{(k)}}$

and $\delta \sigma_{\Re}^{(k)}$

$\delta \sigma_{\Re}^{(k)} \quad \delta \lambda^{(k)} \mathrm{T}^{(k)}: \Psi_{\sigma \Re_{n+1}}^{(k)}$.

Tensor $\mathrm{T}$ can be easily computed considering that the gradient of the derivative of the plastic potential (Eq. (25)) fits with the Hessian of the yield function in $J_{2}$ plasticity

$\frac{\partial \Psi_{\sigma \Re}}{\partial \sigma_{\Re}} \quad \frac{1}{\sigma}\left(\frac{3}{2} I_{d e v} \quad r_{\Re} \otimes r_{\Re}\right)$,

where $r_{\Re}$ is the direction of the inelastic flow in $J_{2}$ plasticity given by the deviatoric tensor
$r_{\Re} \quad \frac{3}{2} \frac{s}{\sigma}$

Thus, applying the Sherman Morrison formula

Т $\quad C \quad \frac{6 G^{2} \Delta \lambda}{3 G \Delta \lambda+\sigma}\left(I_{\text {dev }} \quad \frac{2}{3} r_{\Re} \otimes r_{\Re}\right)$.

Taking into account that the gradient of $f$ is

$\left.\frac{\partial f}{\partial \sigma_{\Re}} \quad \frac{3}{2} \frac{s}{\sigma}+\left[\begin{array}{ll}1 & D\end{array}\right) \alpha+\frac{\mu D}{3}\right] 1$.

Eq. (51) is written as

$\delta \lambda^{(k)} \quad \frac{f_{n+1}^{(k)}}{3 G+\Lambda_{D}}$

with

$\Lambda_{D} \quad 3 K\left(\left(\begin{array}{ll}1 & D_{n+1}\end{array}\right) \alpha+\mu \frac{D_{n+1}}{3}\right)\left(3\left(1 \quad D_{n+1}\right) \alpha_{\Psi}+D_{n+1} \mu_{\Psi}\right)$.

This expression recovers the classical plastic multiplier for Drucker Prager associated plasticity $(k=1)$ when damage is not considered $(D=0)$

$\delta \lambda^{(k)} \frac{f_{n+1}^{(k)}}{3 G+9 K \alpha^{2}}$.

Since the yield surface remains constant during the return, the first subiteration enables us to determine $\Delta \lambda$ and the stress is up dated by Eq. (44) considering the proportionality between $S_{\Re_{n+1}}$ and $s_{\mathfrak{R}_{n+1}}^{\text {trial }}$

$\frac{S_{\Re_{n+1}}}{\sigma_{n+1}} \frac{S_{\Re_{n+1}}^{\text {trial }}}{\sigma_{n+1}^{\text {trial }}}$.

Finally the updated rotated stress is pushed to the spatial configu ration by $\Re_{n+1}$

$$
\begin{aligned}
& \sigma_{n+1} \quad \Delta \mathfrak{R}_{n}^{n+1} \sigma_{\mathfrak{R}_{n}} \Delta \mathfrak{R}_{n}^{n+1^{T}} \\
& +C:\left(\Delta \mathfrak{R}_{n+1 / 2}^{n+1} \Delta t d_{n+1 / 2} \Delta \Re_{n+1 / 2}^{n+1^{T}}\right) \quad \frac{3 G \Delta \lambda}{\sigma_{n+1}^{\text {trial }}} \Re_{n+1} S_{\mathfrak{R}_{n+1}^{\text {trial }}} \mathfrak{R}_{n+1}^{T} \\
& 3 K \Delta \lambda\left[\left(\begin{array}{ll}
1 & D_{n+1}
\end{array}\right) \alpha_{\Psi}+\mu_{\Psi} \frac{D_{n+1}}{3}\right] 1,
\end{aligned}
$$

where the incremental rotation tensors are given by

$\begin{array}{ll}\Delta \mathfrak{R}_{n}^{n+1} & \mathfrak{R}_{n+1} \mathfrak{R}_{n}^{T}, \\ \Delta \mathfrak{R}_{n+1 / 2}^{n+1} & \mathfrak{R}_{n+1} \mathfrak{R}_{n+1 / 2}^{T} .\end{array}$

\subsection{Direct return to the apex}

The singularity of the yield surface (the apex of the cone) re quires a specific treatment that permits to return to it, taking into account that, at this point, trial stress states in a certain region of the stress space will return directly to it. This region is delimited in the Haigh Westergaard space by a cone, coaxial and coincident in the apex with the yield cone, and with generatrixes following the return direction of the return mapping algorithm. The slope of the generatrixes can be determined in the bidimensional space $\{p, \bar{\sigma}\}$ (which is not isomorphous to the stress space) enabling dis crimination between trial states that return with the algorithm described above, and those that return directly to the cone apex.

From the expression of the plastic return $\Delta \sigma_{\Re}^{\text {ret }}$ given by Eq. (44), we can easily determine its spherical and deviatoric components by projecting onto the axes $\{p, \bar{\sigma}\}$ 


$$
\begin{array}{ll}
\Delta p^{\text {ret }} & \left.\frac{\Delta \sigma_{\mathfrak{R}}^{\text {ret }}: 1}{3} \quad 3 K \Delta \lambda\left[\begin{array}{ll}
1 & D_{n+1}
\end{array}\right) \alpha_{\Psi}+\mu_{\Psi} \frac{D_{n+1}}{3}\right] \\
\Delta \sigma^{\text {ret }} & \left(\frac{3}{2} \frac{9 G^{2} \Delta \lambda^{2}}{\left(\sigma_{n+1}^{\text {trial }}\right)^{2}} s_{\mathfrak{R}_{n+1}}^{\text {trial }}: s_{\mathfrak{\Re}_{n+1}}^{\text {trial }}\right)^{1 / 2} \quad 3 G \Delta \lambda
\end{array}
$$

and the slope of the return direction in the bidimensional space $\{p, \bar{\sigma}\}$ is

$$
\kappa^{\text {ret }} \frac{\Delta \sigma^{r e t}}{\Delta p^{r e t}} \frac{G}{K\left[\begin{array}{ll}
(1 & \left.D_{n+1}\right) \alpha_{\Psi}+\mu_{\Psi} \frac{D_{n+1}}{3}
\end{array}\right]} .
$$

Then the trial states inside the region

$$
\sigma_{n+1}^{\text {trial }} \leqslant \kappa^{\text {ret }}\left(p_{n+1}^{\text {trial }} \quad p_{\text {apex }}\right)
$$

with

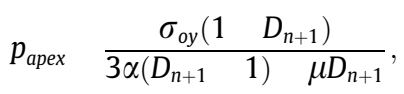

must return directly to the apex (Fig. 2), which corresponds to the hydrostatic stress state

$$
\sigma_{n+1} \quad p_{\text {apex }} 1 \text {. }
$$

\section{Parameter calibration}

The model requires eight parameters, two defining the elastic behavior ( $E$ and $v$ ), three for the yield function $\left(3 \alpha, \sigma_{0 y}\right.$ and $\mu$ ), two for the damage growth $\left(\dot{D}_{o}\right.$ and $\left.\sigma_{0}\right)$, and one for the dilatant effect of the inelastic flow $(k)$. The Young modulus $E$ and Poisson ratio $v$ may be easily found in the literature for ceramic materials used in armor applications [44]. Cohesion $\sigma_{0 y}$ may be related to the tensile yield stress $\sigma_{t}$, a material parameter that may also be easily found in the literature [44], by the expression

$\sigma_{0 y} \sigma_{t}(1+\alpha)$.

The parameters $3 \alpha$ and $\mu$, defining pressure hardening for undam aged and fully fragmented ceramics respectively, may be deter mined, for instance, by fitting curves drawn from confined compression tests $[45,46]$. In our case, these parameters are deter mined by fitting (least squares) the pressure strength curves of the ceramic constitutive model JH2 [13] proposed experimentally by Cronin et al. [47] for different ceramic materials.

Brittle materials fracture when the tensile yield stress $\sigma_{y}$ is reached in a uniaxial tensile test. Thus, the threshold value of the principal stress for the damage growth $\sigma_{1}=\sigma_{0}$ was set equal to $\sigma_{t}$, which also agrees well with the numerical results achieved in the validation of the model. Finally, we have determined the parameters $\dot{D}_{0}$ and $k$ by a simple inverse analysis, using FEM sim ulation and the experimental results provided by Hohler et al. [44]. In this work, the authors analyze the ballistic efficiency of different

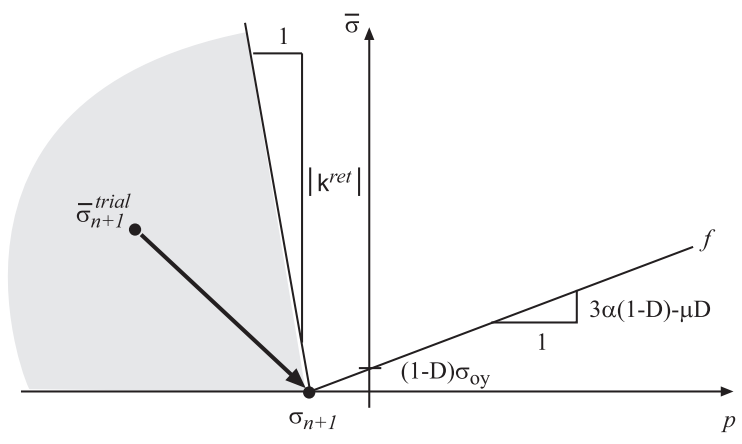

Fig. 2. Direct return to apex for trial stress states subjected to the condition given by Eq. (67). ceramic materials confined by a mild steel box, by means of the depth of penetration (DOP) test, measuring the penetration of a tungsten projectile impacting at $1700 \mathrm{~m} / \mathrm{s}$ against a set of ceramic tiles backed by a block of Rolled Homogeneous Armor (RHA) steel (Fig. 3a). This measure enables us to determine these last two parameters.

\subsection{Numerical simulation}

The finite element code Abaqus Explicit v6.44 [48] was used to simulate the DOP test. Due to the high speed of the process $(v=1700 \mathrm{~m} / \mathrm{s})$ it was considered adiabatic. A structured mesh (Fig. 3b) was developed, using 8 node trilinear displacement and temperature, reduced integration with hourglass control elements. In the impact direction, the characteristic length of the elements of the projectile, the ceramic tiles, and the RHA steel block was $L_{c}=1.5 \mathrm{~mm}$. The elements of the confinement were larger due to the low level of deformation undergone by this part. As boundary conditions, the back side of the RHA steel block was pinned in the impact direction, the mild steel confinement was tied to the block, and an unilateral contact condition was defined between ceramic tiles and confinement. The interactions between the ceramic tiles and RHA steel block, and between the projectile and tiles, were modeled with the general contact kinematic contact algorithm implemented in Abaqus Explicit [48]. Since penetration was expected to occur between the projectile and both the tiles and block, a pure master slave weighting was used in the interaction between these solids. The master surface was all the upper faces of the elements forming the tiles and the steel block, and the slave surface being the projectile nodes. In this way, although certain elements of the head of the projectile and of the target were deleted, the contact between the two parts is maintained through out the penetration process. For the interaction between ceramic tiles, as no penetration was expected, a balanced weighting was used.

For metallic materials used in ballistic applications, elastic strains (and rates) are commonly very small compared to inelastic strains (and rates). Therefore, hypoelastic plastic material models were also employed in this work for the metallic materials, allow ing us to assume the additive decomposition of the rate of defor mation tensor [35]. For these alloys, J2 plasticity, with an isotropic hardening constitutive law was adopted. To take into account the rate dependent behavior and the thermal softening by adiabatic heating that metals undergo in this kind of process, the hardening/softening Johnson Cook law [49] was employed. This is probably the most widely used among those accounting for plastic strain, plastic strain rate, and temperature effects. Since numerous efforts have been made in the past to determine their properties for a large number of metallic materials, it has been implemented in many FE explicit codes. The relation is stated through the following multiplicative equation

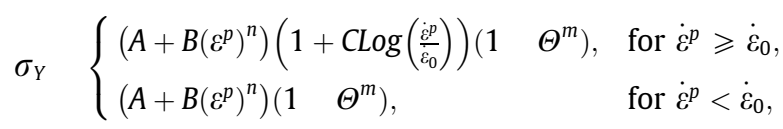

where $\Theta$ is the homologous temperature

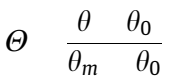

with $\theta, \theta_{0}$ and $\theta_{m}$ being the temperature, the reference temperature, and the melting temperature respectively.

Table 1 shows the values of the material parameters for the metal alloys and their corresponding references. To model the erosion undergone by the projectile, the ceramic tile, and the metal back plate, a simple failure model based on a critical values of the equiv alent plastic strain $\bar{\varepsilon}^{p}$ was used, so that when it reached a critical 


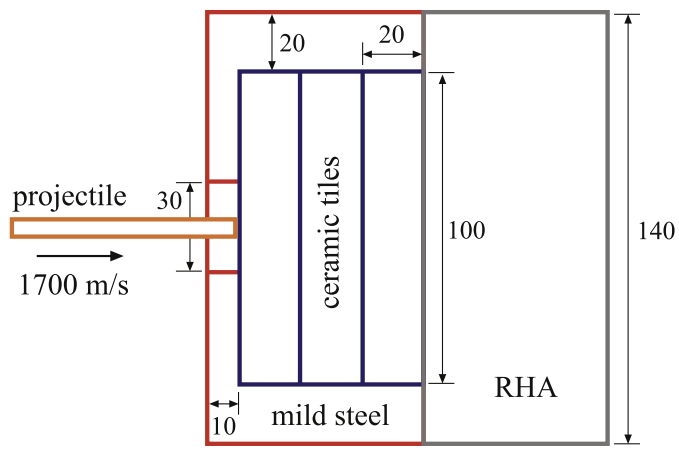

(a)

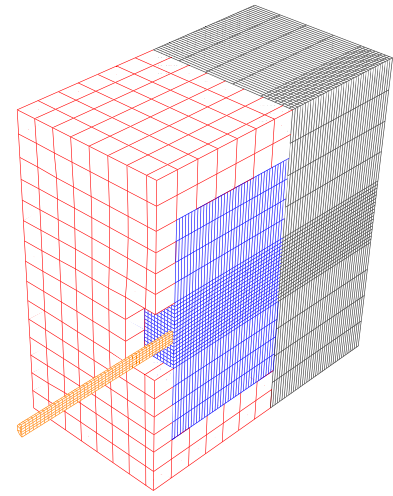

(b)

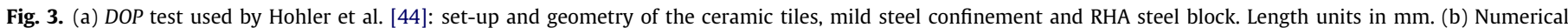
simulation: mesh of the DOP model.

Table 1

Properties of metal alloys used in the simulations.

\begin{tabular}{lccc}
\hline Parameter & $\begin{array}{c}\text { Tungsten alloy } \\
{[50]}\end{array}$ & $\begin{array}{c}\text { RHA steel } \\
{[51]}\end{array}$ & $\begin{array}{c}\text { Mild steel } \\
{[52]}\end{array}$ \\
\hline$E(\mathrm{GPa})$ & 345 & 207 & 200 \\
$v$ & 0.33 & 0.3 & 0.3 \\
Density $\left(\mathrm{kg} / \mathrm{m}^{3}\right)$ & 17,600 & 7800 & 7870 \\
$A(\mathrm{MPa})$ & 1093 & 1240 & 532 \\
$B(\mathrm{MPa})$ & 1270 & 509 & 229 \\
$n$ & 0.42 & 0.26 & 0.3 \\
$\mathrm{C}$ & 0.0188 & 0.014 & 0.027 \\
$\varepsilon_{0}\left(\mathrm{~s}^{1}\right)$ & 1 & 1 & 1 \\
$m$ & 0.78 & 1.03 & 1 \\
$\theta_{0}\left({ }^{\circ} \mathrm{C}\right)$ & 25 & 25 & 25 \\
$\theta_{m}\left({ }^{\circ} \mathrm{C}\right)$ & 1580 & 1430 & 1520 \\
Specific heat $\left(\mathrm{J} \mathrm{kg}{ }^{1} \mathrm{~K}^{1}\right)$ & 134 & 500 & 481 \\
Conductivity $\left(\mathrm{W} \mathrm{m}{ }^{1} \mathrm{~K}{ }^{1}\right)$ & 45 & 44.5 & 44.5 \\
Thermal exp. coef. & 4.3 & 1.3 & 1.3 \\
$\quad\left({ }^{\circ} \mathrm{C}{ }^{1}\right) \cdot 10{ }^{5}$ & & & \\
Quinney-Taylor coef. & 0.7 & 0.9 & 0.85 \\
\hline
\end{tabular}

Table 2

Properties of high-purity alumina for the proposed model.

\begin{tabular}{lc}
\hline Parameter & Value \\
\hline$E(\mathrm{GPa})$ & 378 \\
$v$ & 0.22 \\
$3 \alpha$ & 0.65 \\
$\mu$ & 0.21 \\
$\sigma_{0 y}(\mathrm{MPa})$ & 292 \\
$\sigma_{0}(\mathrm{MPa})$ & 240 \\
$D_{0}(\mathrm{~Pa} \mathrm{~s})$ & $8 \cdot 10^{3}$ \\
$k$ & 0.7 \\
\hline
\end{tabular}

value $\varepsilon_{\text {failure }}$, the element was deleted from the model. For the cera mic $\bar{\varepsilon}^{p}$ was defined as

$$
\begin{aligned}
\varepsilon^{p} & \int_{0}^{t} \dot{\varepsilon}^{p} d t \quad \int_{0}^{t} \sqrt{\frac{2}{3}} d_{\Re}^{p}: d_{\Re}^{p} d t \\
& \left.\int_{0}^{t} \dot{\lambda} \sqrt{1+2\left[\left(\begin{array}{ll}
1 & D
\end{array}\right) \alpha_{\Psi}+\mu_{\Psi} \frac{D}{3}\right.}\right]^{2} d t .
\end{aligned}
$$

Certainly the element deletion technique causes an artificial loss of mass and momentum in the system. However, the model accurately reproduces classical experimental results in the field of impact mechanics, as will be shown in the validation of the model.
Table 3

Thicknesses of alumina-aluminium lightweight protections and impact velocities for each protection.

\begin{tabular}{lllc}
\hline Alumina thickness $(\mathrm{mm})$ & Aluminium thickness $(\mathrm{mm})$ & $V_{1}(\mathrm{~m} / \mathrm{s})$ & $V_{2}(\mathrm{~m} / \mathrm{s})$ \\
\hline 8.1 & 4 & 786 & 829 \\
8.1 & 6 & 815 & 916 \\
8.1 & 8 & 995 & 1091 \\
\hline
\end{tabular}

Table 4

Properties of metal alloys employed in the validation simulations.

\begin{tabular}{lcc}
\hline Parameter & Aluminium 6061-T6 & AISI 4340 steel \\
& {$[53]$} & {$[54]$} \\
\hline$E(\mathrm{GPa})$ & 73 & 208 \\
$v$ & 0.33 & 0.3 \\
Density $\left(\mathrm{kg} / \mathrm{m}^{3}\right)$ & 2705 & 7850 \\
$A(\mathrm{MPa})$ & 335 & 1150 \\
$B(\mathrm{MPa})$ & 85 & 739 \\
$n$ & 0.11 & 0.26 \\
$C$ & 0.012 & 0.014 \\
$\varepsilon_{0}\left(\mathrm{~s}^{1}\right)$ & 1 & 1 \\
$m$ & 1 & 1.03 \\
$\theta_{0}\left({ }^{\circ} \mathrm{C}\right)$ & 25 & 25 \\
$\theta_{m}\left({ }^{\circ} \mathrm{C}\right)$ & 600 & 1520 \\
Specific heat $\left(\mathrm{J} \mathrm{kg}{ }^{1} \mathrm{~K}{ }^{1}\right)$ & 963 & 500 \\
Conductivity $\left(\mathrm{W} \mathrm{m}{ }^{1} \mathrm{~K}{ }^{1}\right)$ & 170 & 44.5 \\
Thermal exp. coef. & 2.2 & 1.3 \\
$\quad\left({ }^{\circ} \mathrm{C}{ }^{1}\right) \cdot 10{ }^{5}$ & & 0.8 \\
Quinney-Taylor coef. & & \\
\hline
\end{tabular}

Sixteen simulations were carried out, varying both $\dot{D}_{0}$ and $k$ un til the expected value for residual penetration was reached. Table 2 summarizes the final values of the parameters for high purity alumina.

\section{Model validation}

The model was validated with the experimental results of Den Reijer [5] for impacts on alumina aluminum armor. This author developed a flash radiography system to measure the position of the projectile and the armor during the penetration process. The tests were performed for three different configurations of the armor, and two impact velocities for each configuration: $V_{1}$ corre sponding to projectile arrest, and $V_{2}$ corresponding to armor perfo ration. The main characteristics of the tests are summarized in Table 3. 

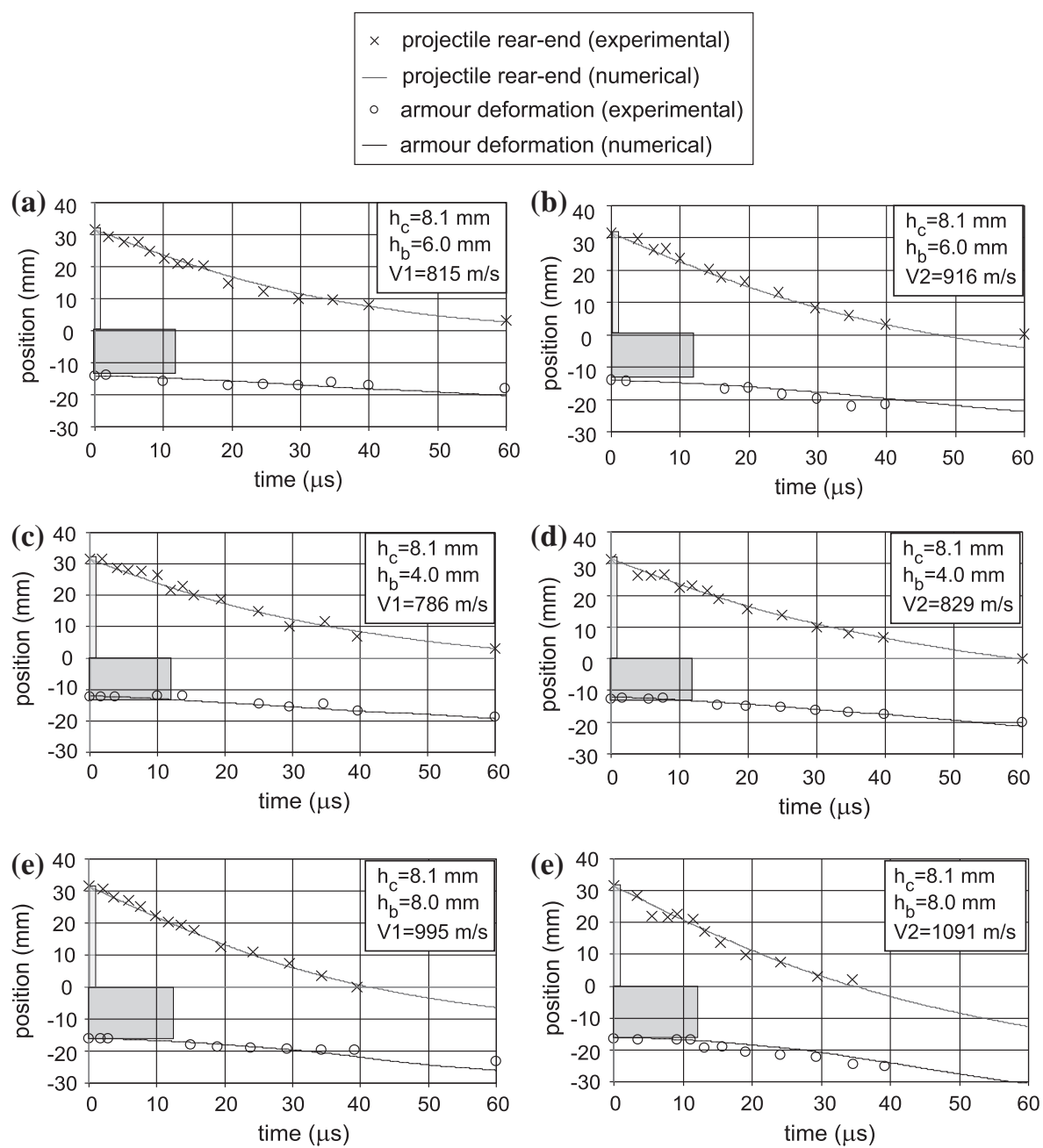

Fig. 4. Numerical and experimental [5] position vs. time curves of the projectile rear-end and of the armor back side. $h_{c}=$ alumina thickness, $h_{b}=$ aluminum thickness.

The aspects of the numerical simulation were discussed in Section 4.1. Free boundary conditions were established due to the short duration of the process $(60 \mu \mathrm{s})$ and an initial condition of velocity in the impact direction was imposed on the nodes of the projectile. The values of the material parameters for the 6061 T6 aluminium (backing plate) and AISI 4340 steel (projec tile), and their corresponding references are shown in Table 4. For the high density alumina, the parameters shown in Table 2 were used.

Fig. 4 shows the position of the projectile rear end and the dis placement of the armor back side for experimental and numerical results. The model predicts these variables with accuracy for both complete perforation and projectile arrest.

\section{Conclusions}

A simple constitutive model to reproduce the behavior of cera mic materials used in armor applications was presented. The mod el considers fragmentation of the ceramic tile through a damage parameter for which growth is driven by the maximum principal stress. The yield stress is assumed to depend on pressure and dam age, and inelastic flow is governed by a non associated law to reduce the dilatant effect. The parameters of the model can be eas ily determined for different ceramics used in armor protection. Also, a semi implicit time integration algorithm been developed to integrate the proposed constitutive model. The algorithm is easily implemented and inherits the robustness and stability of return mapping schemes. The performance of the model and the algorithm is evaluated by comparing its results with those of classical experiments; such comparisons show that the numerical results agree quantitatively with the experimental ones.

\section{Acknowledgements}

The authors are indebted to the Spanish Ministry of Education (Project DPI2008 06408/DPI), and to the Region of Madrid (Project CCG08 UC3M/MAT 4464) for the financial support.

\section{Appendix A. Overall implementation scheme for the co- rotational configuration}

(1) Elastic predictor

$$
\sigma_{\mathfrak{\Re}_{n+1}}^{\text {trial }} \quad \sigma_{\mathfrak{R}_{n}}+C:\left(\Delta \varepsilon_{\mathfrak{R}}\right)
$$

(2) Check the yield condition

$$
\begin{aligned}
& \text { If } f\left(\sigma_{\Re_{n+1}^{\text {trial }}}^{\text {th }}, D_{n+1}\right)<0 \Rightarrow \\
& \sigma_{\Re_{n+1}} \quad \sigma_{\Re_{n+1}}^{\text {trial }} \\
& D_{n+1} \quad D_{n} \\
& \epsilon_{\mathfrak{R}_{n+1}}^{p} \quad \epsilon_{\mathfrak{R}_{n}}^{p} \\
& \text { end } \\
& \text { Else } \Rightarrow \text { go to } 3
\end{aligned}
$$


(3) Choice of plastic return

$$
\begin{aligned}
& \text { If } \sigma_{n+1}^{\text {trial }} \leqslant \kappa^{\text {ret }} \cdot\left(\begin{array}{ll}
p_{n+1}^{\text {trial }} & \left.p_{\text {apex }}\right) \Rightarrow \text { go to } 4 \\
\text { Else } \Rightarrow \text { go to } 5
\end{array}\right.
\end{aligned}
$$

(4) Direct return to apex

$$
\begin{array}{llll}
\sigma_{\mathfrak{\Re}_{n+1}} & p_{\text {apex }} 1 & & \\
D_{n+1} & D_{n}+\dot{D}_{o}\left\langle\sigma_{1 n}\right. & \left.\sigma_{o}\right\rangle \Delta t \\
\epsilon_{\mathfrak{R}_{n+1}}^{p} & \epsilon_{\mathfrak{R}_{n}}^{p}+\Delta \epsilon_{\mathfrak{R}^{p}}^{p} & \epsilon_{\mathfrak{R}_{n}}^{p} & C^{1}:\left(\begin{array}{lll}
\sigma_{\mathfrak{R}_{n+1}} & \sigma_{\mathfrak{R}_{n}}
\end{array}\right) \\
\text { end } & & &
\end{array}
$$

\section{(5) Return mapping algorithm}

$$
\begin{aligned}
& \Delta \lambda \frac{f_{n+1}}{3 G+3 K\left(\left(\begin{array}{ll}
1 & \left.\left.D_{n+1}\right) \alpha+\mu \frac{D_{n+1}}{3}\right)\left(3\left(\begin{array}{ll}
1 & D_{n+1}
\end{array}\right) \alpha_{\Psi}+D_{n+1} \mu_{\Psi}\right.
\end{array}\right)\right.} \\
& \sigma_{\Re_{n+1}} \quad \sigma_{\Re_{n+1}}^{\text {trial }} \quad \Delta \lambda\left(3 G \frac{S_{\Re_{n+1}}}{\sigma_{n+1}}+3 K\left[\left(\begin{array}{ll}
1 & D_{n+1}
\end{array}\right) \alpha_{\Psi}+\mu_{\Psi} \frac{D_{n+1}}{3}\right] 1\right) \\
& D_{n+1} \quad D_{n}+\dot{D}_{o}\left\langle\sigma_{1 n} \quad \sigma_{o}\right\rangle \Delta t \\
& \epsilon_{\mathfrak{R}_{n+1}}^{p} \quad \epsilon_{\mathfrak{R}_{n}}^{p} \quad C^{1}:\left(\begin{array}{ll}
\sigma_{\mathfrak{R}_{n+1}} & \sigma_{\mathfrak{R}_{n}}
\end{array}\right) \\
& \text { end. }
\end{aligned}
$$

\section{References}

[1] Wilkins, ML. Third progress report on light armor program. Tech. rep., Lawrence Radiation Laboratory, University of California, Livermore, USA; 1964.

[2] Florence A. Interaction of projectiles and composite armour. Part II. Menlo Park, California, USA: Stanford Research Institute; 1969

[3] Wilkins ML. Calculation of elastic-plastic flow. Met Comp Phys 1964;3:211-62.

[4] Mayseless M, Goldsmith W, Virostek SP, Finnegan SA. Impact on ceramic targets. J Appl Mech 1987;54:373-8.

[5] Den Reijer P. Impact on ceramic faced armour. Ph.D. thesis, Delf University of Technology; 1991.

[6] Zaera R, Sánchez-Gálvez V. Modelling the fracture processes in the ballistic impact on ceramic armours. J Phys IV 1997;7(3):687-92.

[7] Woodward R. A simple one-dimensional approach to modelling ceramic composite armour defeat. Int J Impact Eng 1990;9(4):455-74.

[8] Zaera R, Sánchez-Sáez S, Pérez-Castellanos JL, Navarro C. Modelling of the adhesive layer in mixed ceramic/metal armours subjected to impact. Compos Part A: Appl Sci Manuf 2000;31(3):823-33.

[9] López-Puente J, Arias A, Zaera R, Navarro C. The effect of the thickness of the adhesive layer on the ballistic limit of ceramic/metal armours. An experimental and numerical study. Int J Impact Eng 2005;32(1-4):321-36.

[10] Heard $\mathrm{HC}$, Cline CF. Mechanical-behavior of polycrystalline $\mathrm{BeO}, \mathrm{Al}_{2} \mathrm{O}_{3}$ and $\mathrm{AlN}$ at high-pressure. J Mater Sci 1980;15:1889-97.

[11] Curran DR, Seaman L, Shockey DA. Dynamic failure of solids. Phys Report $1987 ; 147: 253-388$

[12] Johnson G, Holmquist T. A computational constitutive model for brittle materials subjected to large strains, high strain rates and high pressures. In: Meyers LEMMA, Staudhammer KP, editors. Shock-wave and high-strain-rate phenomena in materials. NY: Marcel Dekker Inc.; 1992. p. 1075-81.

[13] Johnson G, Holmquist T. An improved computational constitutive model for brittle materials. In: Schwartz GSMRSC, Shaner JW, editors. High-pressure science and technology, American institute of physics conference. NY: AIP Press; 1993. p. 981-4.

[14] Fahrenthold E. A continuum damage model for fracture of brittle solids under dynamic loading. J Appl Mech 1991;58:904-9.

[15] Cortés R, Navarro C, Martínez MA, Rodríguez J, Sánchez-Gálvez V. Numerical modelling of normal impact on ceramic composite armours. Int J Impact Eng 1992;12:639-51.

[16] Rajendran A. Modeling the impact behavior of ad85 ceramic under multiaxial loading. Int J Impact Eng 1994;15:749-68.

[17] Rajendran AM, Grove D. Modeling the shock response of silicon carbide, boron carbide and titanium diboride. Int J Impact Eng 1996;18:611-31.

[18] Liu H, Rajendran HZDA, Atluri S. Computational modeling of impact response with the RG damage model and the meshless local Petrov-galerkin (MLPG) approaches. Comput Mater Continua 2006;4:43-54

[19] Simha H, Bless S, Bedford A. Computational modeling of the penetration response of a high-purity ceramic. Int J Impact Eng 1991;27:65-86.

[20] Bar-on E, Rubin M, Yankelevsky D. Thermomechanical constitutive equations for the dynamic response of ceramics. Int J Solids Struct 2003;40:4519-48.
[21] Zuo Q, Addessio F, Dienes J, Lewis M. A rate-dependent damage model for brittle materials based on the dominant crack. Int J Solids Struct 2006;43:3350-80.

[22] Deshpande V, Evans A. Inelastic deformation and energy dissipation in ceramics: a mechanism-based constitutive model. J Mech Phys Solids 2008;56:3077-100.

[23] Camacho G, Ortiz M. Computational modeling of impact damage in brittle materials. Int J Solids Struct 1996;33:2899-938.

[24] Zhou F, Molinari J. Stochastic fracture of ceramics under dynamic tensile loading. Int J Solids Struct 2004;41:6573-96.

[25] Zhou F, Molinari J. On the rate-dependency of dynamic tensile strength of a model ceramic system. Comput Methods Appl Mech Eng 2005;194:1693-709.

[26] Lee M, Kim E, Yoo Y. Simulation of high speed impact into ceramic composite systems using cohesive-law fracture model. Int J Impact Eng 2008;35:1636-41.

[27] Malvar LJ, Crawford JE, Morrill KB. A plasticity concrete material model for dyna3D. Int J Impact Eng 1997;19:847-73.

[28] Zhou XQ Hao H. Mesoscale modelling and analysis of damage and fragmentation of concrete slab under contact detonation. Int J Impact Eng 2009;36:1315-26.

[29] Hentz S, Donzé FV, Daudeville L. Discrete element modelling of concrete submitted to dynamic loading at high strain rates. Comput Struct 2004;82:2509-24.

[30] Riedel W, Thoma K, Hiermaier S. Penetration of reinforced concrete by BETA-B500 numerical analysis using a new macroscopic concrete model for hydrocodes. In: Ninth international symposium on the interaction of the effect of munitions with structures. Berlin-Strausberg, Germany; 1999, pp. 315-322.

[31] Riedel W. Beton unter dynamischen lasten meso- und makromechanische modelle und ihre parameter. Ph.D. thesis, Institut Kurzzeitdynamik, ErnstMach-Institut, Freiburg; 2000

[32] Tu Z, Lu Y. Evaluation of typical concrete material models used in hydrocodes for high dynamic response simulations. Int J Impact Eng 2009;36:132-46.

[33] Kröner E. Allgemeine kontinuumstheorie der versetzungen und eigenspannungen. Arch Rational Mech Anal 1959;4:273-334.

[34] Lee EH. Elastic-plastic deformation at finite strains. J Appl Mech 1969;36:1-6.

[35] Nemat-Nasser S. On finite deformation elastoplasticity. Int J Solids Struct 1982;18:857-72.

[36] Khan A, Huang S. Continuum theory of plasticity. NY: John Wiley \& Sons; 1995.

[37] Curran D, Seaman L, Cooper T, Shockey D. Micromechanical model for comminution and granular flow of brittle material under high strain rate application to penetration of ceramic targets. Int J Impact Eng 1993;13(1):53-83.

[38] Denoual C, Hild F. Dynamic fragmentation of brittle solids: a multi-scale model. Eur J Mech A/Solids 2002;21:105-20.

[39] Jirásek M, Bazănt Z. Inelastic analysis of structures. England: John Wiley \& Sons; 2002.

[40] Zaera R, Fernández-Sáez J. An implicit consistent algorithm for the integration of thermoviscoplastic constitutive equations in adiabatic conditions and finite deformations. Int J Solids Struct 2006;43(6):1594-612.

[41] Simó J, Hughes T. Computational Inelasticity. NY: Springer-Verlag; 1998.

[42] Doghri I. Mechanics of deformable solids. Berlin: Springer-Verlag; 2000.

[43] Hagege B. Simulation du comportement mécanique des renforts fibreux en grandes transformations: application aux renforts tricotés. Ph.D. thesis, ENSAM, Paris, 2004

[44] Hohler V, Stilp A, Weber K. Ranking methods of ceramics and experimental optimisation of a laminated target with ceramics. In: Proceedings of the lightweight armour system symposium. Cranfield: Royal Military College of Science; 1995.

[45] Forquin P, Arias A, Zaera R. An experimental method of measuring the confined compression strength of geomaterials. Int J Solids Struct 2007:44:4291-317.

[46] Chocron S, Walker J, Nicholls A, Dannemann K, Anderson C. Analytical model of the confined compression test used to characterize brittle materials. J Appl Mech 75.

[47] Cronin D, Bui K, Kaufmann C, McIntosch G, Berstad T. Implementation and validation of the Johnson-Holmquist ceramic material model in LS-Dyna. In: GmbH D, editor. 4th European LS-dyna users conference, vol. D. Alemania: Ulm; 2003. p. 47-60.

[48] HKS, Abaqus explicit v6.4 user's manual, version 6.4 edition, ABAQUS Inc. Richmond, USA; 2003.

[49] Johnson GR, Cook WH. A constitutive model and data for metals subjected to large strains, high strain rates, and temperatures. In: Proceedings of the 7th international symposium Ballistics, The Hague, The Nederlands; 1983, pp. 1-7.

[50] Zhigang W, Jilin Y, Jianrong L, Yongchi L, Shisheng H. Influence of stress condition on adiabatic shear localization of tungsten heavy alloys. Int J Impact Eng 2001;26:843-52.

[51] Gee D, Levinson RWGS. Blast phenomena associated with high-speed impact. Int J Impact Eng 2007;34(2):178-88.

[52] Myungsoo P, Jeonghoon Y, Dong-Teak C. An optimization of a multi-layered plate under ballistic impact. Int J Solids Struct 2005;42:123-37.

[53] Dabboussi W, Nemes JA. Modeling of ductile fracture using the dynamic punch test. Int J Mech Sci 2005;47(8):1282-99.

[54] Banerjee B. The mechanical threshold stress model for various tempers of AISI 4340 steel. Int J Solids Struct 2007;44(3-4):834-59. 